\title{
Best co-approximation on 2-fuzzy metric space
}

\author{
Thangaraj Beaula ${ }^{1 *}$ and P. Jayasingh Manohar ${ }^{2}$
}

\begin{abstract}
In this paper the concept of best coapproximation in 2-fuzzy metric space is introduced. Various concepts like coproximinal, co-chebyshev, translation invariant, orthogonal on 2-fuzzy metric linear spaces are established. Using these concepts some related theorems and lemmas are developed.

Keywords

Co-Chebyshev, coproximinal, coapproximation.

AMS Subject Classification 54A40, 54E35, 54H 25.

1,2 P.G and Research Department of Mathematics, TBML College, Porayar-609307, India.

${ }^{*}$ Corresponding author: ${ }^{1}$ edwinbeaula@yahoo.co.in

Article History: Received 21 December 2018; Accepted 11 February 2019

(C)2019 MJM.
\end{abstract}

\section{Contents}

1 Introduction 37

2 Preliminaries 37

3 Best Coapproximation on 2-Fuzzy Metric space... 38

4

Conclusion

References 39

\section{Introduction}

The concept of fuzzy sets introduced by L.A.Zadeh in 1965[19] became active in the field of research. Among other fields, a progressive development is made in the field of fuzzy topology. One of the most important problems in fuzzy topology is to obtain an appropriate concept of fuzzy metric space. This problem has been investigated by many different authors in different view point.

In 1975, Kramosil and Michalek[5] introduced the concept of fuzzy metric space. George and Veeramani[4] modified the concept of fuzzy metric space. In 1974, K.C.Wong[18] defined fuzzy point and discussed some topological properties. Zike Dong[2] defined pseudo-metric spaces with metric defined between fuzzy points rather than between fuzzy sets. The concept of fuzzy metric space with metric was defined between fuzzy points by Nai-Hung Hsu[10].

A new kind of approximation, called best coapproximation was introduced in normed linear spaces by C.Franchetti and M.Furi[3]. The theory of best coapproximation is much less developed as compared to the theory of best approximation in abstract spaces.
The purpose of this paper is to discuss the existence and uniqueness results on best approximation and best coapproximation in two-fuzzy metric linear spaces.

\section{Preliminaries}

Definition 2.1. [4] A binary operation $*:[0,1] \times[0,1] \rightarrow$ $[0,1]$ is called a $t$-norm if for all $a, b, c, d \in[0,1]$ the following conditions are satisfied

(i) $a * 1=a$.

(ii) $a * b=b * a$.

(iii) $a * b \leq c * d$ whenever $a \leq c, b \leq d$.

(iv) $a *(b * c)=(a * b) * c$.

Definition 2.2. [16] The 3-tuple $(X, M, *)$ is called a fuzzy metric space if $X$ is an arbitrary set, $*$ is a continuous $t$-norm and $M$ is a fuzzy set in $X^{2} \times[0, \infty]$ satisfying the conditions

(M1) $M(x, y, t)=0$.

(M2) $M(x, y, t)=1, \forall t>0$ if and only if $x=y$.

(M3) $M(x, y, t)=M(y, x, t)$.

(M4) $M(x, y, t) * M(y, z, s) \leq M(x, z, t+s)$

(M5) $M(x, y,):.[0, \infty] \rightarrow[0,1]$ is left continuous.

(M6) $\lim _{t \rightarrow \infty} M(x, y, t)=1$. 
Definition 2.3. [6] The 3-tuple $(X, M, *)$ is called a 2-fuzzy metric space if $X$ is an arbitrary set, $*$ is a continuous $t$-norm and $M$ is a fuzzy set in $X^{2} \times[0, \infty]$ satisfying the conditions

(2-M1) $M(f, g, t)=0$.

(2-M2) $M(f, g, t)=1, \forall t>0$ if and only if $f=g$.

(2-M3) $M(f, g, t)=M(g, f, t)$.

(2-M4) $M(f, g, t) * M(g, h, s) \leq M(f, h, t+s)$

(2-M5) $M(f, g,):.[0, \infty] \rightarrow[0,1]$ is left continuous.

(2-M6) $\lim _{t \rightarrow \infty} M(f, g, t)=1$.

\section{Best Coapproximation on 2-Fuzzy Metric space}

Definition 3.1. Let $(\mathscr{F}(X), M, *)$ be a 2-fuzzy metric space and $A$ be a nonempty subset of $\mathscr{F}(X)$. An element $f_{0} \in A$ is said to be the coapproximation to $f \in \mathscr{F}(X)$ if

$$
M\left(f_{0}, g, t\right) \geq M(f, g, t) \text { for all } g \in \mathscr{F}(X)
$$

The set of all best coapproximations to $f_{0} \in A$ is denoted by $\mathscr{F} R_{A}(f)$.

The set $A$ is called coproximinal if $\mathscr{F} R_{A}(f)$ contains atleast one $g \in A$ for every $f \in \mathscr{F}(X)$. Iffor each $f \in \mathscr{F}(X), \mathscr{F} R_{A}(f)$ has exactly one $g \in A$, then $A$ is said to beco-chebyshev. A 2-fuzzy metric space $(\mathscr{F}(X), M, *)$ is said to be convex if $M\left(\lambda f_{1}+(1-\lambda) f_{2}, g, t\right) \geq \min \left\{M\left(f_{1}, g, t\right), M\left(f_{2}, g, t\right)\right\}$ for all $f_{1}, f_{2} \in \mathscr{F}(X)$ and $\lambda \in(0,1)$

A 2-fuzzy metric $M$ is said to be translation invariant if $M\left(f+f_{0}, g+g_{0}, t\right)=M(f, g, t)$ for all $f, g, f_{0} \in \mathscr{F}(X)$.

Definition 3.2. A 2-fuzzy metric linear space is a 2-fuzzy metric space with fuzzy translation invariant 2-fuzzy metric provided

(i) $M(f+g, t) \geq \min \{M(f, t), M(g, t)\}$ for all $f, g \in \mathscr{F}(X)$

(ii) $M(-f, t) \geq M(f, t)$

(iii) $M(k x, t) \geq \min \{N(k, t), M(f, t)\}$ for $k \in \mathscr{F}$ (the field)

(iv) $N(1, t) \geq M(\overline{0}, t)$ where $N: K \times(0, \infty) \rightarrow[0,1]$ and $K \subset$ $F$ the field.

Let $(\mathscr{F}(X), M, *,+,$.$) be a 2-fuzzy metric linear space and$ $f, g \in \mathscr{F}(X)$ we say that $f$ is orthogonal to $g$, if $M(f, \overline{0}, t) \geq$ $M(f, \alpha g, t)$ for every scalar $\alpha$. It is denoted by $f \perp g$.

Further $A$ is orthogonal to $g$ if $g \perp f$ for every $f \in \mathscr{F}(X)$.

Theorem 3.3. Let $A$ be a 2-fuzzy linear subspace of the 2fuzzy metric linear space $(\mathscr{F}(X), M, *)$ such that $A$ is orthogonal to $\left(f-g_{0}\right)$ theng $_{0} \in \mathscr{F} R_{A}(f)$.
Proof. Given A is orthogonal to $\left(f-g_{0}\right)$ where $f \in \mathscr{F}(X)$ and $g_{0} \in \mathscr{F}\left(R_{A}(f)\right)$ then $g$ is orthogonal to $\left(f-g_{0}\right)$ for every $g \in A$.

(ie) $M(g, \overline{0}, t) \geq M\left(g, \alpha\left(f-g_{0}\right), t\right)$ for every $\alpha$.

Suppose $\alpha=1$ then $M(g, \overline{0}, t) \geq M\left(g, f-g_{0}, t\right)$ for every $g \in A$.

As $M$ is fuzzy translation invariant

$$
\begin{gathered}
M\left(g+g_{0}, \overline{0}+g_{0}, t\right) \geq M\left(g+g_{0}, f-g_{0}+g_{0}, t\right) \\
\text { (ie) } M\left(g_{0}, g+g_{0}, t\right) \geq M\left(f, g+g_{0}, t\right)
\end{gathered}
$$

satisfying the requirements that $g_{0} \in \mathscr{F} R_{A}(f)$.

Theorem 3.4. Let $A$ be a 2-fuzzy linear subspace of a two fuzzy metric linear space $(\mathscr{F}(X), M, *,+,$.$) and g_{0} \in A$. Then $\alpha g_{0} \in \mathscr{F} R_{A}(\alpha f)$ for every scalar $\alpha$ if and only if $A$ is orthogonal to $\left(f-g_{0}\right)$.

Proof. Assume $\alpha g_{0} \in \mathscr{F} R_{A}(\alpha f)$.

Then $M\left(\alpha g_{0}, g, t\right) \geq M(\alpha f, g, t)$ for every $g \in A$.

(ie) $M\left(\alpha g_{0}-\alpha g_{0}, g-\alpha g_{0}, t\right) \geq M\left(\alpha f-\alpha g_{0}, g-\alpha g_{0}, t\right)$ for every $g \in A$

(ie) $M\left(\overline{0}, g-\alpha g_{0}, t\right) \geq M\left(\alpha f-\alpha g_{0}, g-\alpha g_{0}, t\right)$

(ie) $M\left(\overline{0}, g^{\prime}, t\right) \geq M\left(\alpha f-\alpha g_{0}, g^{\prime}, t\right)$ for every $g^{\prime} \in A$

Hence $\mathrm{A}$ is orthogonal to $f-g_{0}$.

Conversely, suppose A is orthogonal to $f-g_{0}$ then $g^{\prime}$ is orthogonal to $f-g_{0}$ for every $g^{\prime} \in A$.

(ie) $M\left(g^{\prime}, \overline{0}, t\right) \geq M\left(g^{\prime}, \alpha\left(f-g_{0}\right), t\right)$

(ie) $M\left(g^{\prime}+\alpha g_{0}, \alpha g_{0}, t\right) \geq M\left(g^{\prime}+g_{0} \alpha+\alpha\left(f-g_{0}\right)+\alpha g_{0}, t\right)$

(ie) $M\left(\alpha g_{0}, g^{\prime}+\alpha g_{0}, t\right) \geq M\left(\alpha f, g^{\prime}+\alpha g_{0}, t\right)$

(ie) $M\left(\alpha g_{0}, g, t\right) \geq M(\alpha f, g)$ for every $g \in A$ and scalar.

Therefore $\alpha g_{0}^{\prime} \in \mathscr{F} R_{A}(\alpha f)$ satisfies the requirement.

Lemma 3.5. Let $A$ be a 2-fuzzy closed linear subspace of a 2-fuzzy metric linear space $(\mathscr{F}(X), M, *,+,$.$) . If f$ is not an element of $A$ such that $\alpha f$ has a best coapproximation in $A$ then every element of the subspace $\{x, A\}$ has a best coapproximation in A.

Proof. Consider $\alpha f+g^{\prime} \in f, A$ and $g_{0} \in \mathscr{F} R_{A}(\alpha f)$ then $M\left(g_{0}, g, t\right) \geq M(\alpha f, g, t)$

$$
\begin{aligned}
& \Rightarrow M\left(g_{0}+g^{\prime}, g+g^{\prime}, t\right) \geq M\left(\alpha f+g^{\prime}, g+g^{\prime}, t\right) \text { for every } \\
& g \in G \\
& \Rightarrow M\left(g_{0}+g^{\prime}, g^{\prime \prime}, t\right) \geq M\left(\alpha f+g^{\prime}, g^{\prime \prime}\right) \text { for every } g^{\prime \prime} \in G
\end{aligned}
$$

therefore $g_{0}+g^{\prime} \in \mathscr{F} R_{A}\left(\alpha f+g^{\prime}\right)$.

Thus every element of the subspace $\{f, A\}$ has a best coapproximation in A. 
Lemma 3.6. Let $A, B$ be 2-fuzzy subspaces of 2-fuzzy metric linear space $(\mathscr{F}(X), M, *,+,$.$) so that A$ is a subset of $B$. If an element $f$ not in $B$ has the best coapproximation in $B$ and if every element of $B$ has the best coapproximation in $A$ then $f$ has the best coapproximation in A.

Proof. Let $f \in \mathscr{F}(X)$ such that it does not belong to B, so that $k_{0} \in \mathscr{F} R_{B}(f)$

Then $M\left(k_{0}, k, t\right) \geq M(f, k, t)$ for every $k \in B$.

for $k_{0} \in B$, let $g_{0} \in A$ be so that, $M\left(g_{0}, g, t\right) \geq M\left(k_{0}, g, t\right)$

then $M\left(g_{0}, g, t\right) \geq M(f, g, t)$ for every $g \in A$ and thus $g_{0} \in$ $\mathscr{F} R_{A}(f)$.

Note 3.7. Using lemma3.5 and lemma3.6 we deduce the following theorem.

Theorem 3.8. Let $A$ be a 2-fuzzy subspace of a 2-fuzzy metric space $(\mathscr{F}(X), M, *,+,$.$) , if there exists at least one element$ $f \in \mathscr{F}(X) \backslash A$ such that $\alpha f$ has the best coapproximation in $A$ then for any subspace A of $\mathscr{F}(X)$ every element of $\mathscr{F}(X)$ has a best approximation in $A$.

Theorem 3.9. Let $A$ be a 2-fuzzy coproximinal subspace of a 2-fuzzy metric linear space $(\mathscr{F}(X), M, *,+,$.$) if \mathscr{F} R_{A}^{-1}(\overline{0})$ is a convex set then $A$ is co-chebyshev.

Proof. Let $f \in \mathscr{F}(X)$ and $g_{1}, g_{2} \in \mathscr{F} R_{A}(f)$.

Take $f-g_{1}=h^{\prime}$ and $f-g_{2}=h^{\prime \prime}$ where $f-g_{1}, f-g_{2} \in$ $\mathscr{F} R_{A}(\overline{0})$

then $M(0, g, t) \geq M\left(f-g_{1}, g, t\right) \forall g \in A$

Since $\overline{0} \in \mathscr{F} R_{A}\left(f-g_{1}\right)$,

$$
\begin{gathered}
M\left(g_{1}, g+g_{1}, t\right) \geq M\left(f, g+g_{1}, t\right) \\
\text { (ie) } \quad M\left(g_{1}-f, g+g_{1}-f, t\right) \geq M\left(0, g+g_{1}-f, t\right) \\
\text { (ie) } \quad M\left(g_{1}-f, g^{\prime}, t\right) \geq M\left(0, g^{\prime}, t\right)
\end{gathered}
$$

Where $g^{\prime}=g+g_{1}-f \in A$ therefore $g_{1}-f$ belongs to $\mathscr{F} R_{A}(\overline{0})$. Suppose $\mathscr{F} R_{A}^{-1}(\overline{0})$ is convex and $f-g_{2}, g_{1}-f$ are elements in $\mathscr{F} R_{A}^{-1}(\overline{0})$

$$
\begin{aligned}
M\left(\lambda\left(f-g_{2}\right)+(1-\lambda)\left(g_{1}-f\right), g, t\right) & \geq \min \left\{M\left(f-g_{2}, g, t\right),\right. \\
& \left.M\left(g_{1}-f, g, t\right)\right\} \\
& \geq \min \{M(\overline{0}, g, t), M(\overline{0}, g, t)\} \\
& =M(\overline{0}, g, t)
\end{aligned}
$$

Which implies $\lambda\left(f-g_{2}\right)+(1-\lambda)\left(g_{1}-f\right) \in \mathscr{F} R_{A}^{-1}(\overline{0})$ If $\lambda=1 / 2$ then $\left(g_{1}-g_{2}\right) / 2 \in \mathscr{F} R_{A}^{-1}(\overline{0})$ and also $\left.\left(g_{1}-g_{(} 2\right)\right) / 2$ belongs to A,

it is obvious that $\mathscr{F} R_{A}^{-1}(\overline{0}) \cup A=\{\overline{0}\}$ and $\operatorname{sog}_{1}=g_{2}$ and so A is co-chebyshev.

Theorem 3.10. If $A$ is co-chebyshev 2-fuzzy subspace of a 2fuzzy metric space $(\mathscr{F}(X), M, *)$ then the graph of the 2-fuzzy metric coprojection $\mathscr{F} R_{A}$ is 2-fuzzy closed.

Proof. The 2-fuzzy metric co projection $\mathscr{F} R_{A}: \mathscr{F}(X) \rightarrow$ $F\left(2^{\mathscr{F}} R_{A}\right)$ where $\mathscr{F}\left(2^{\mathscr{F} R_{A}}\right)$ is the set of all fuzzy subsets of $\mathscr{F}\left(R_{A}\right)$ and the graph is defined as

$$
G\left(\mathscr{F} R_{A}\right)=\left\{\left(f, \mathscr{F} R_{A}(f)\right) / f \in \mathscr{F}(X)\right\}
$$

Let $(f, h)$ be the limit point of $G\left(\mathscr{F} R_{A}\right)=\left\{\left(f, \mathscr{F} R_{A}(f)\right) / f \in\right.$ $\mathscr{F}(X)\}$ there exists a sequence $\left(f_{n}, \mathscr{F} R_{A}\left(f_{n}\right)\right)$ in $G\left(\mathscr{F} R_{A}\right)$ such that $\left(f_{n}, \mathscr{F} R_{A}\left(f_{n}\right)\right) \rightarrow(f, h)$. That is $f_{n} \rightarrow f$ and $\mathscr{F} R_{A}\left(f_{n}\right)$ $\rightarrow h$.

It is obvious that $M\left(\mathscr{F} R_{A}\left(f_{n}\right), g, t\right) \geq M\left(f_{n}, g, t\right)$ for every $g \in A$,

Then $M(h, g, t) \geq M(f, g, t)$ for every $g \in A$ and so $h \in \mathscr{F} R_{A}(f)$. Since A is co-chebyshev $\mathscr{F} R_{G}(f)$ contains only one point $\mathrm{h}$ and so $\mathscr{F} R_{G}(f)=\{h\}$. Hence $(f, h)$ belongs to $G\left(\mathscr{F} R_{A}\right)$ and so it is 2-fuzzy closed.

\section{Conclusion}

In the present study, we introduced the concept of best co approximation in 2-fuzzy metric space, which generalized various concepts like coproximinal, co-Chebyshev translation invariant, orthogonal on 2- fuzzy metric linear spaces. We also proved some related theorems and lemmas.

\section{References}

[1] Cheney E.W., Introduction to Approximation Theory, McGraw Hill, New York, 1966.

[2] Dong Z., Fuzzy Pseudo-Metric Spaces, J. Math. Anal. Appl., 86(1982), 74-95.

[3] Franchetti C., Furi M., Some Characteristic Properties of Hilbert Spaces, Rev. Roumaine Math. Pure Appl., 17(1972), 1045-1048.

[4] George A., Veeramani P., On Some Results in Fuzzy Metric Space, Fuzzy Sets and Syst., 64(1994), 395-399.

[5] Kramosil O., Michalek J., Fuzzy Metric and Statistical Metric Spaces, Kybernetika, 11(1975), 326-334.

[6] Kumar S., Common Fixed Point Theorem in Fuzzy 2Metric Spaces, Uni. Din. Bacau. Studiisiceretirisciintifice, Serials: Mathematical, 18(2008), 111-116.

[7] Mazaheri H., MaalekGhaini F.M., Quasi-Orthogonality of the Best Approximate Sets, Nonlinear Anal., 65(2006), 534-537.

[8] Mazaheri H., Modaress S.M.S., Some Results Concerning Proximinality and Co Proximinality, Nonlinear Anal., 62(2005), 1123-1126.

[9] MuthukumarS., A Note on Best and Best Simultaneous Approximation, Indian J. Pure Appl. Math., 11(1980), 715-719.

[10] Nai-Hung Hsu, On Completion of Fuzzy Metric Spaces, Bull. Nat. Taiwan Nor. Univ., 37(1992), 385-392.

[11] Narang T.D., Best Approximation in Metric Spaces, Publ. Sec. Mat. Uni. Autonoma Barcelona, 27(1983), 71-80.

[12] Narang T.D., Best Approximation in Metric Linear Spaces, Math. Today, 5(1987), 21-28.

[13] Narang T.D., Singh S.P., Best Coapproximation in Metric Linear Spaces, Tamkang J. Math., 30(1999), 241-252.

[14] Papini P.L., Singer I., Best Coapproximation in Normed Linear Spaces, Monatsh. Math., 88(1979), 27-44. 
[15] Rao K., Chandrasekhara, Functional Analysis, Narosa Publishing House, New Delhi, 2002.

[16] Sharma S., On Fuzzy Metric Space, South East Asian Bulletin of Mathematics, 26(2002), 133-145.

[17] Singer I, Best Approximations in Normed Linear Spaces by Elements of Linear Sub-Spaces, Springer-Verlag, New York, 1970.

[18] Wong C.K., Fuzzy Points and Local Properties of Fuzzy Topology, J. Math. Anal. Appl., 46(1974), 316-328.

[19] Zadeh L.A., Fuzzy Sets, Information and Control, 8(1965), 338-353.

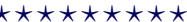

ISSN(P):2319-3786

Malaya Journal of Matematik

ISSN(O):2321 - 5666

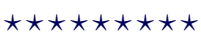

